\title{
Enterococcal Prosthetic Joint Infection Treated With Prosthesis Retention: A Case Report
}

\author{
Corinne Lahoud ${ }^{\mathrm{a}, \mathrm{b}}$, Souheil Hallit ${ }^{\mathrm{a}, \mathrm{c}, \mathrm{f}}$, Firas El-Masrid, \\ Matta Matta ${ }^{\text {d, e }}$, Rabih Hallit ${ }^{\text {a, b, e }}$
}

\begin{abstract}
Periprosthetic joint infection may occur after prosthetic joint replacement. Treatment usually requires removal of the prosthesis in addition to systemic antibiotics. We report a case of enterococcal prosthetic knee infection that was treated with arthroscopic lavage and intravenous antibiotics (ampicillin $2 \mathrm{~g}$ every $4 \mathrm{~h}$ for 6 weeks and gentamicin $80 \mathrm{mg}$ every $8 \mathrm{~h}$ for 2 weeks) with retention of the prosthesis. Upon discharge, the patient was placed on oral amoxicillin $1 \mathrm{~g}$ twice daily for a duration of 2 years. The patient now has been off antibiotics for 3 years, and is showing no signs of recurrence of his infection on his last follow-up.
\end{abstract}

Keywords: Prosthetic joint; Infection; Enterococcus

\section{Introduction}

Septic arthritis (SA) refers to an infection of a joint, most commonly due to a bacterial infection. In most cases, the patient presents with acute joint pain, swelling, warmth, and restricted movement. The most commonly involved joint (50\% of cases) is the knee. Other frequently involved joints are the wrists, ankles, and hips [1]. Although the causative organisms are multiple, Staphylococcus aureus (SA) (including methicillinresistant Staphylococcus aureus (MRSA)) remains the most common bacterium infecting adult joints. Multiple risk factors were identified for SA, including the presence of a prosthetic joint. Periprosthetic joint infection (PJI) occurs in 1 to 2 per-

Manuscript submitted May 13, 2018, accepted June 12, 2018

aFaculty of Medicine and Medical Sciences, Holy Spirit University of Kaslik (USEK), Jounieh, Lebanon

botre Dame des Secours Hospital University Hospital Center, Byblos City, Lebanon

'Research Department, Psychiatric Hospital of the Cross, Jal Eddib, Lebanon ${ }^{\mathrm{d}}$ Faculty of Medicine, Saint-Joseph University, Beirut, Lebanon

eInfectious Diseases Department, Bellevue Medical Center, Mansourieh, Lebanon

${ }^{f}$ Corresponding Author: Souheil Hallit, Street 8, Building 560, 1st floor, Biakout, Lebanon. Email: souheilhallit@hotmail.com

doi: https://doi.org/10.14740/jmc3089w cent of joint replacement surgeries. The risk of PJI is greater for knee arthroplasty than hip arthroplasty $[1,2]$.

\section{Case Report}

We present a case of a 75-year-old male who presented to the hospital with a right swollen painful knee along with fever. The patient had undergone total knee replacement 4 years prior to presentation for osteoarthritis. The patient was admitted and systemic antibiotics with vancomycin $1 \mathrm{~g}$ intravenous (IV) Q12 $\mathrm{h}$ and ceftriaxone $2 \mathrm{~g}$ IV daily were started empirically after cultures were taken. The patient underwent arthroscopic lavage of the knee with retention of the prosthesis. Blood cultures as well as articular fluid cultures grew Enterococcus faecalis sensitive to ampicillin. The patient was switched to IV ampicillin and gentamicin. A trans-esophageal echocardiogram showed no evidence of endocarditis and subsequent blood cultures were negative. A colonoscopy revealed the presence of a malignant colon mass that was subsequently removed surgically at a later stage. The patient received ampicillin $2 \mathrm{~g}$ every $4 \mathrm{~h}$ for 6 weeks and gentamicin $80 \mathrm{mg}$ every $8 \mathrm{~h}$ for 2 weeks with a favorable clinical and biological response. Upon discharge, he was placed on oral amoxicillin $1 \mathrm{~g}$ twice daily for a duration of 2 years. The patient now has been off antibiotics for 3 years, and is showing no signs of recurrence of his infection on his last follow-up.

\section{Discussion}

PJIs present a major therapeutic challenge for both healthcare providers and patients. The treatment of PJI usually includes both surgery and antimicrobial therapy. Surgical options include resection arthroplasty with reimplantation (in one or two stages), resection arthroplasty in the absence of subsequent hardware reimplantation, debridement and retention of prosthesis or amputation. A study done on 645 hips and 1,145 knees compared the different treatment strategies of PJIs. The two-stage protocol therapy was found to have the lowest risk for both PJI recurrence and need for additional surgery, making it the method of choice [3].

The timing of appearance of the infection is important in guiding the choice of treatment: for instance, prosthesis removal is required in the majority of delayed-onset and late- 
onset PJI.

DAIR refers to a regimen of debridement, antibiotics, irrigation, and retention of the prosthesis. This method of treatment has shown to be usually effective for acute PJI when there is a well-fixed prosthesis without a sinus tract. It is curative in up to $71 \%$ of cases. It is also done in patients for whom surgery is contraindicated, but with a higher risk of relapse [2].

Several studies showed the effect of prosthesis retention on PJI. A retrospective study done on 91 patients showed that $66 \%$ were infection free at a 3-year follow-up [4]. Two other retrospective studies showed suppressive antibiotic therapy to be a good alternative in patients who were not candidates for revision surgery. This was proven for infections caused by Staphylococcal and Streptococcal pathogens [5, 6]. Moreover, two large multicenter studies (including more than 340 patients each) followed up PJI managed with DAIR, one involving Streptococcus sp., the other involving Staphylococcus sp., both showed a failure of approximately 45\% [7-8]. Nevertheless, one of the studies proved that the exchange of removable components, the early use of rifampin and long treatment with $\beta$-lactams, were independent factors of success prediction [7].

Following initial antibiotic therapy for retained PJI, indefinite antibiotic suppression with an oral antimicrobial agent may be warranted. Studies showed that for treatment of staphylococcal PJI managed with debridement and component retention, initial therapy consists of 2 to 6 weeks of pathogenspecific IV therapy in combination with rifampin (300 to 450 $\mathrm{mg}$ orally twice daily or $600 \mathrm{mg}$ once daily). Subsequent therapy for knee PJI consists of 6 months of pathogen-specific oral therapy in combination with rifampin (vs. 3 months for hip, elbow, shoulder or ankle PJI). For treatment of retained PJI due to organisms other than $S$. aureus, initial therapy consists of 4 to 6 weeks of pathogen-specific IV or oral therapy with high bioavailability [2].

As noted above, most available studies were mainly done on Staphylococcus and Streptococcus species. A retrospective study done between 1999 and 2014 on 87 patients with enterococcal PJI showed an overall treatment success in 45 patients. Only $39.4 \%$ were treated by irrigation and debridement. This study stated that enterococcal PJI has a high rate of treatment failure and that combination antibiotic therapy seems to have better outcomes [9].

Two retrospective studies on PJI due to Enterococcus species treated with DAIR were done in January 2015. Duijf et al collected a sample of 44 patients with an early PJI $(<$ 3 months after the implantation) with Enterococcus sp. The mean follow-up was 678 days. The success rate was of $66 \%$, as the prosthesis was retained in 29 patients. As for the antibiotic regimen, patients were either treated with teicoplanin $400 \mathrm{mg}$, rifampicin $450 \mathrm{mg}$, vancomycin or amoxicillin $500 \mathrm{mg}$ or a combination of these antibiotics for 3 months postoperatively [10].

Tornero et al collected a sample of 94 patients, where only early PJIs were included ( $<90$ days from index surgery). The mean follow-up period was also 678 days. The success rate was between 62 and $66 \%$ which was considered far from the $88 \%$ success rate obtained in staphylococcal infections treated with DAIR and a combination of levofloxacin and rifampin. At the end of this study, the author stated the importance of finding a fixed antibiotic regimen for PJI due to Enterococcus species [11].

Our patient had an enterococcal prosthetic knee infection that occurred 4 years after the prosthesis was placed. Despite the late onset PJI, we elected to retain the prosthesis and attempt arthroscopic lavage along with aggressive IV antibiotics followed by 2 years of oral amoxicillin. The decision to stop the antibiotics was difficult given the risk of recurrence of the infection but after a long discussion with the patient, and given the fact that he was completely asymptomatic for 2 years with inflammatory markers (ESR, CRP) within normal range all along, the decision was made to stop the oral antibiotics with a close and careful follow-up. The patient has been off oral antibiotics for 3 years now with no signs of recurrence of the infection.

\section{Conflict of Interest}

The authors have no conflict of interest to report.

\section{Funding Support}

None.

\section{References}

1. Don L Goldenberg, MDDaniel J Sexton M. Septic arthritis in adults - UpToDate. https:/www-uptodate-com. ezproxy.usek.edu.lb/contents/septic-arthritis-in-adults? search $=$ prostheticsepticarthritis \&source $=$ search_result $\&$ selectedTitle $=3 \sim 150 \&$ usage_type $=$ default\&display_ rank=3. Accessed March 15, 2018.

2. Elie Berbari, MD, FIDSA Larry M Baddour, MD, FIDSA F. Prosthetic joint infection: Epidemiology, clinical manifestations, and diagnosis - UpToDate. https:// www-uptodate-com.ezproxy.usek.edu.lb/contents/ prosthetic-joint-infection-epidemiology-clinical-manifestations-and-diagnosis? search=prostheticsepticarthr itis\&source $=$ search_result\&selectedTitle $=2 \sim 150 \&$ usa ge type $=$ default\&display_rank=2. Accessed March 17, 2018.

3. Gallo J, Smizansky M, Radova L, Potomkova J. [Comparison of therapeutic strategies for hip and knee prosthetic joint infection]. Acta Chir Orthop Traumatol Cech. 2009;76(4):302-309.

4. Kuiper JW, Vos SJ, Saouti R, Vergroesen DA, Graat HC, Debets-Ossenkopp YJ, Peters EJ, et al. Prosthetic jointassociated infections treated with DAIR (debridement, antibiotics, irrigation, and retention): analysis of risk factors and local antibiotic carriers in 91 patients. Acta Orthop. 2013;84(4):380-386.

5. Wouthuyzen-Bakker M, Nijman JM, Kampinga GA, van Assen S, Jutte PC. Efficacy of antibiotic suppressive therapy in patients with a prosthetic joint infection. $\mathrm{J}$ Bone $\mathrm{Jt}$ Infect. 2017;2(2):77-83. 
6. Prendki V, Zeller V, Passeron D, Desplaces N, Mamoudy P, Stirnemann J, Marmor S, et al. Outcome of patients over 80 years of age on prolonged suppressive antibiotic therapy for at least 6 months for prosthetic joint infection. Int J Infect Dis. 2014;29:184-189.

7. Lora-Tamayo J, Senneville E, Ribera A, Bernard L, Dupon M, Zeller V, Li HK, et al. The Not-So-Good prognosis of streptococcal periprosthetic joint infection managed by implant retention: the results of a large multicenter study. Clin Infect Dis. 2017;64(12):1742-1752.

8. Lora-Tamayo J, Murillo O, Iribarren JA, Soriano A, Sanchez-Somolinos M, Baraia-Etxaburu JM, Rico A, et al. A large multicenter study of methicillin-susceptible and methicillin-resistant Staphylococcus aureus prosthet- ic joint infections managed with implant retention. Clin Infect Dis. 2013;56(2):182-194.

9. Kheir MM, Tan TL, Higuera C, George J, Della Valle CJ, Shen M, Parvizi J. Periprosthetic joint infections caused by enterococci have poor outcomes. J Arthroplasty. 2017;32(3):933-947.

10. Duijf SV, Vos FJ, Meis JF, Goosen JH. Debridement, antibiotics and implant retention in early postoperative infection with Enterococcus sp. Clin Microbiol Infect. 2015;21(5):e41-42.

11. Tornero E, Soriano A. Prosthetic joint infection due to Enterococcus sp treated with debridement, antibiotics and retention of the implant (DAIR). Clin Microbiol Infect. 2015;21(5):e43-44. 